\title{
Towards an Interpretation of Intestinal Motility Using Capsule Endoscopy Image Sequences
}

\author{
Hai $\mathrm{Vu}^{1}$, Tomio Echigo ${ }^{2}$, Ryusuke Sagawa ${ }^{1}$, Keiko Yagi $^{3}$, Masatsugu Shiba ${ }^{4}$, \\ Kazuhide Higuchi ${ }^{4}$, Tetsuo Arakawa ${ }^{4}$, and Yasushi Yagi ${ }^{1}$ \\ 1 The Institute of Scientific and Industrial Research, Osaka University \\ \{vhai, sagawa, yagi\}@am. sanken.osaka-u.ac.jp \\ ${ }^{2}$ Osaka Electro-Communication University \\ echigo@isc.osakac.ac.jp \\ ${ }^{3}$ Kobe Pharmaceutical University \\ k-yagi@kobepharma-u.ac.jp \\ ${ }^{4}$ Graduate School of Medicine, Osaka City University \\ \{shiba, khiguchi, arakawat\}@med.osaka-cu.ac.jp
}

\begin{abstract}
Human intestinal motility is presented by the propagation of peristaltic waves with their frequencies gradually decreasing along the length of the small bowel. This paper describes a heuristic method, which can be used towards interpreting intestinal motility through recognizing their frequency characteristics from capsule endoscopy image sequences. First, image features that reflect peristaltic activities are extracted to build a functional signal. Then, a Multi-Resolution Analysis technique in the wavelet domain is used to decompose the functional signal taking into account the non-stationary nature of intestinal motility. For peristaltic waveform recognition, the method relies on the principle of peak detections from the decomposed signals. Each waveform is detected when it exceeds a baseline level. The frequency characteristics are interpreted through analysis of the waveform appearance and their velocity propagation. Three healthy sequences were tested in experiments. The estimated trends of the peristaltic wave propagation from the experimental results show a frequency gradient, which follows the well-recognized characteristics of intestinal motility propagation. Therefore, this study is the first demonstration of a detailed interpretation of intestinal motility, and we suggest that further research focuses on intestinal motility dysfunctions.
\end{abstract}

\section{Introduction}

Interpretation of human intestinal motility has been the subject of exhaustive research over many decades by physiologists. The intestinal motility consists of peristaltic waves (slow waves) 12], in which the propagation of waveforms along the length of the small bowel has also been well recognized and confirmed in the literature, e.g. 123. There is a gradient in the propagation velocity of the peristaltic waves, where the frequency of appearance of waveforms is highest in the proximal regions and lowest in the distal regions; for example, in man the maximal frequency is 12 cycles/min in the duodenum and $3-5$ cycles $/ \mathrm{min}$ in

T. Wada, F. Huang, and S. Lin (Eds.): PSIVT 2009, LNCS 5414, pp. 839-850, 2009.

(C) Springer-Verlag Berlin Heidelberg 2009 
the terminal ileum [123]. Although the intestinal motility characteristics can be affected by disorders such as bacterial overgrowth, intestine obstruction or paralysis [1|2, the clinical applications of these findings are still limited because it is necessary to take many recordings along the entire length of the small bowel. The recent recording technique [4] is still an invasive measurement because of the long distance and loop configuration of the small bowel.

Capsule Endoscopy (CE) [5] was recently introduced as a non-invasive and effective means of inspecting bleeding regions, Crohn's disease and suspected tumors in the small bowel [6]7. The CE utilizes a swallowable endoscopic device that is ingested and propelled by peristaltic waves through the GastroIntestinal (GI) tract. Although this technique was not originally designed for assessment of intestinal motility, CE image sequences present a useful source of information for investigating intestinal motility (e.g. works in 8 1011]). Thus the aim of this study was to use a functional signal extracted from the CE image sequences to interpret intestinal motility. This is a promising method for overcoming the limitations of the current techniques because of its non-invasive nature and minimal demands on physicians' time. To investigate intestinal motility using CE image sequences, works in 91011] proposed methods to detect intestinal contractions. They used the same approaches that utilized the learning schemes to recognize the contractions. The contractile patterns can be represented by star-wise patterns [9, linear radial patterns [10] or as a directional histogram of edge intestinal folds [1]. Although the results of these studies show successful recognition rates, it is still unclear what information can be derived from results of the detected contractions to understand intestinal motility. On the other hand, from the viewpoint of GI physiology [12], locating only the positions of the contractions does not seem to provide enough information to interpret intestinal motility. Thus, in contrast with the earlier approaches, we attempted to use a heuristic method, which measured the propagation of the peristaltic waves instead of identifying the contractile patterns.

We first considered the disparity of consecutive frames in a CE image sequence such as color dissimilarity, motion displacement, and changes in edges of the intestinal folds. This step can be considered a series of intestinal motility observations. Based on the high correlation of these features, a functional signal is built by averaging multiple observations to reduce artifacts or other influencing factors. Then, the signal is decomposed into its components at different scales by wavelet transformation taking into account the non-stationary characteristics of intestinal motility. The attractive properties of decomposition signals are that they reflect the noise level and baseline level, which form the waveforms of the peristaltic waves. For waveform recognition, the method relies on the principle of peak detection of the decomposed signal where each waveform exceeds a baseline level. The propagation of slow waves are influenced by the frequency appearance of the peaks and their velocity. The method was tested on sequences obtained from three healthy volunteers. Estimated trends of slow wave propagation along the $\mathrm{CE}$ and transit time determined from the experimental results matched the well-known frequency gradient of intestinal motility. This study was the first to 
provide a detailed interpretation of intestinal motility and that the results can be used to identify motility dysfunctions in patients.

\section{Developing a Functional Signal of Intestinal Motility from CE Image Features}

\subsection{Intestinal Motility and Its Characteristics}

Intestinal motility is manifested by electrical activity, that constitutes electrical oscillations called slow waves 2]. Schematic views of the waveforms (convex and concave waveforms) are shown in Fig. 1a. With intermittent bursts of rapid electrical oscillations, spike bursts occur and are superimposed on the slow waves signal (Fig. 1b). The spike bursts are associated with muscular contractions that produce the segmentary contractions (Fig. 1c). The characteristics of slow waves include the appearance or propagation velocity of these waveforms. As stated by 112 , the propagation velocity decreases in a series of steps from proximal to distal regions of the small bowel, as shown in Fig. 1d. For further detail, please refer to Chapter 7 in [1] or Chapter 6 in [2].

It can be noted that the concave and/or convex waveforms are always present, even in the absence of contractions. For recognition of the contractile patterns from the CE image sequences, the contractions in Fig. 1C are well described in

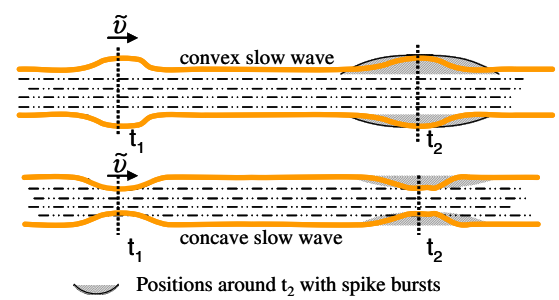

(a) Propagation of the slow wave

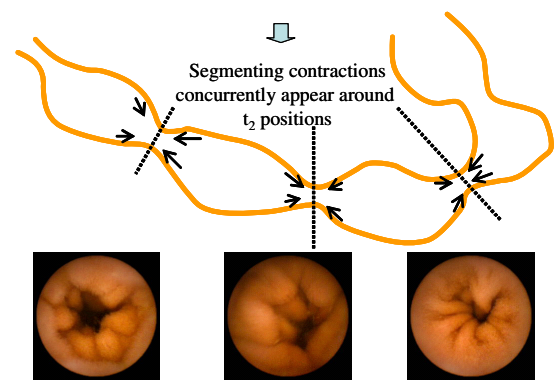

(c) Segmenting contractions

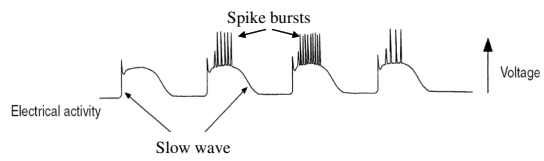

(b) Spike bursts superimposed on the slow wave signal

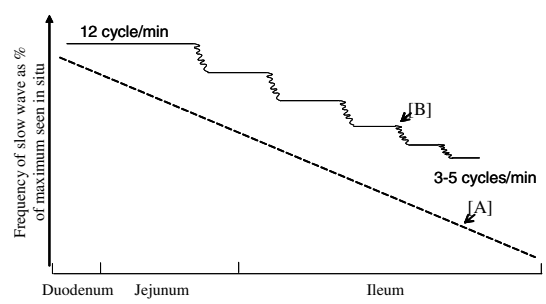

(d) A gradient frequency for slow wave propagation

Fig. 1. Organization and frequency characteristics of intestinal motility 
the earlier studies [91011], whereas the concave/convex patterns are ambiguously described. In fact, the propagation of slow waves propel the CE through the small bowel and, therefore, this represents the main factor responsible for the changes between the acquired images. These changes can be measured by identifying the disparity between consecutive frames. Although this measurement can be affected by other factors such as human activities during examinations or imminent movements (or non-rigidity) of the small intestine. From the view of signal processing, an ensemble averaging technique has advances as it eliminates undesirable noise through multiple observations. To develop a functional signal of intestinal motility from CE image sequences, these observations can be derived from image features that are high correlated.

\subsection{Developing the Functional Signal from Image Features}

In a typical examination, CE takes approximately 7-8 hours to pass through the GI tract and captures images at a rate of $2 \mathrm{fps}$. Therefore, an image sequence has around 57,000 frames, including 20,000 to 30,000 frames that belong to the small bowel regions, which can be used to investigate intestinal motility. To measure the disparity between adjacent frames, we considered a series of image features that were successfully used in previous studies. These features include the edges of intestinal folds (in 891011] for recognizing the segmentary contractions), color dissimilarity and motion displacements (in [12] for evaluating image disparity).

The CE image features include a standard size of 256x256 pixels, 8 bit per channel in RGB color space 13. To determine color dissimilarity between frames $\langle n, n+1\rangle(\operatorname{Dissim}(n))$, we used the color local histogram method in [14]. The image is divided into blocks, with the number of blocks $N_{\text {blocks }}=64$. The color histogram is applied to each block by dividing RGB components into $N_{\text {bins }}=16$. The distance of local histograms is calculated by $L 1$ distance. $\operatorname{Dissim}(n)$ is obtained by accumulating the different blocks. The motion displacement (Motion $(n)$ ) is evaluated as the length of the maximum motion vector, in which the motion fields of frames $\langle n, n+1\rangle$ are detected and tracked using the Kanade-Lucas-Tomasi (KLT) algorithm [15 16 17]. The Canny operator techniques [18] was used to identify the edges of intestinal folds. Edge pixels were counted in a region where most of the edges appear. $\operatorname{Edge}(n)$ is calculated by subtracting the edge pixels detected in frames $\langle n, n+1\rangle$. Feature extractions of a typical sequence including 50 frames are shown in Fig. 2.

The extracted signals are normalized into $[0,1]$, with the maximum value indicating the most different and the minimum value indicating the best match of two consecutive frames. To evaluate the correlation of these signals, we calculated their energy in a duration $\Delta T$. Figure 3 shows the energy of the extracted signals with $\Delta T=1 \mathrm{~min}$. of a full sequence. This figure shows the high correlations of the selected features. Therefore, the functional signal of intestinal motility was calculated by:

$$
S(n)=\frac{\operatorname{Dissim}(n)+\operatorname{Motion}(n)+\operatorname{Edge}(n)}{3}
$$



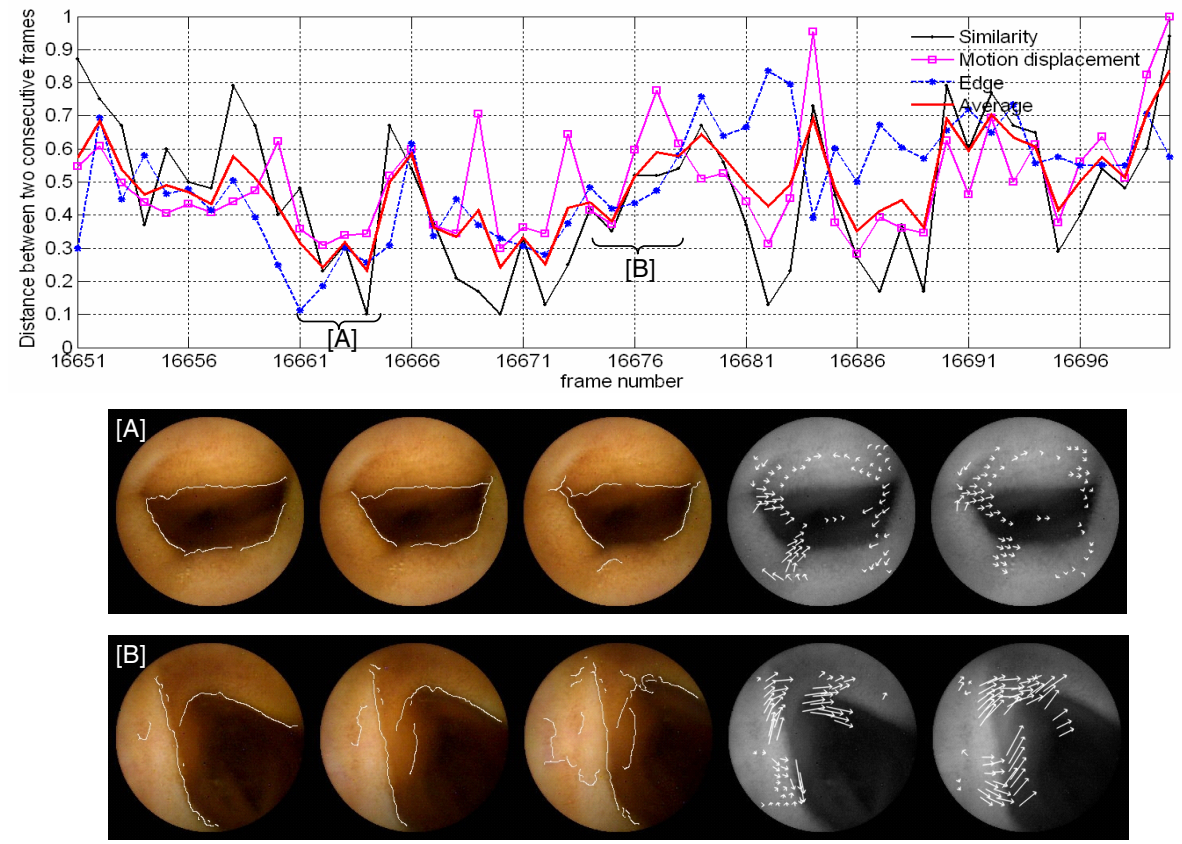

Fig. 2. The feature extractions of a typical sequence including 50 frames. Each bottom panel shows image features around the corresponding positions; the first three images show the edge detections. Results are superimposed on the original images. The next two images show the motion displacements between them.

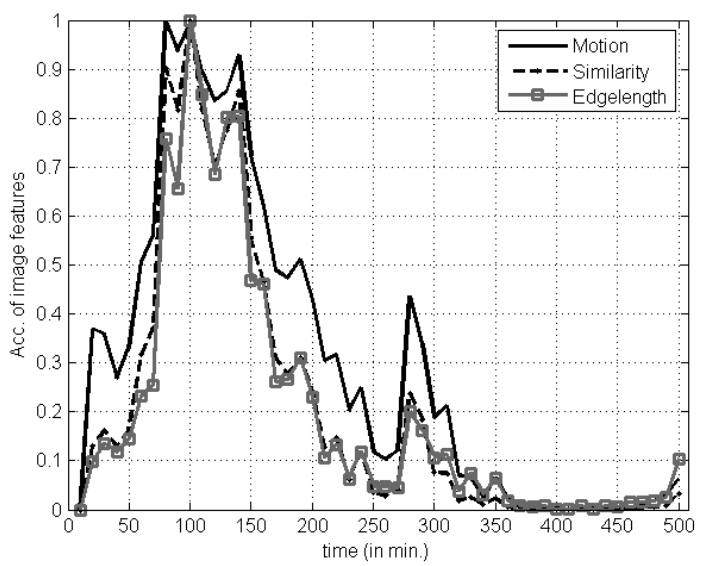

Fig. 3. Accumulating energy of the extracted signals within 1 minute of a full sequence 


\section{Multi-resolution Analysis and Waveform Detection from Decomposition Signals}

\subsection{Multi-resolution Analysis Technique}

Similar to other non-stationary biosignals such as ECG and EGG, the functional signal $S(n)$ is affected by different conditions such as the subject's health condition, natural characteristics of intestinal motility. To exploit their properties such as frequency-time, Multi-Resolution Analysis (MRA) techniques are commonly used because the scale-based nature of the MRA effectively separates the components of the signal into pieces that can be subjected to further analysis. In this study, it is meaningful if the contents of the $S(n)$ signal such as noise and the waveforms of slow waves can be determined from the decomposed signals.

Of the available MRA methods, wavelet transformation is a common used technique. The signal was decomposed using a wavelet orthonormal function (for the details, please refer to Chapter 4 in [19]). Hereafter, we discuss two practical considerations that are the choice of mother wavelets and the decomposition level. Irrespective of the mathematical properties of the mother wavelet, a basic requirement is that it looks similar to the patterns we want to localize in the signal. As is well described in studies [1|2|3|20|21], the waveforms have an approximately triangular shape with some harmonics. Therefore, the Daubechies wavelet $d b 3$ configuration was selected. The decomposition level $(J)$ is selected so that the center frequency at each scale can cover the frequency appearance of waveforms. As described in Sec 2 , the predetermined value $J=6$ is considered a reasonable value because at the capturing rate at $2 \mathrm{fps}$ (or a sampling period $T=0.5(s))$, the frequency at each scale can drop in a range from 2.5 cycles $/ \mathrm{min}$ at level 6,10 cycles/min at level 3 to 20 cycles/min at level 1 . The MRA decomposes the signal $S(n)$ into the detail $D_{j}$ and smoothing $A_{j}$ components, with $j$ is a level from 1 to $J=6$. Figure 4 shows the decomposed signals of an original signal including 1200 frames (in 10 minutes). The properties of MRA analysis ensure the energy preserving condition:

$$
\|E\|^{2}=\sum_{j=1}^{J=6} D_{j}^{2}+S_{6}^{2}
$$

where $\|E\|^{2}$ is the energy of the signal, $\sum D_{j}^{2}$ is the energy of the detail signals and $S_{6}^{2}$ is the energy of the smoothing component at level 6 .

\subsection{Recognizing the Waveforms from the Decomposed Signals}

In studies 2021], intestinal motility was measured by electrical devices, such as strain gauge transducers. The local contractions are denoted by a triangular shape on the output signals with several criteria. For example, there is a baseline level where the threshold voltage is exceeded so that the beginning of the contraction can be recognized; or the duration of these contractions exceed a predetermined value. Based on these observations, we considered that a smoothing 


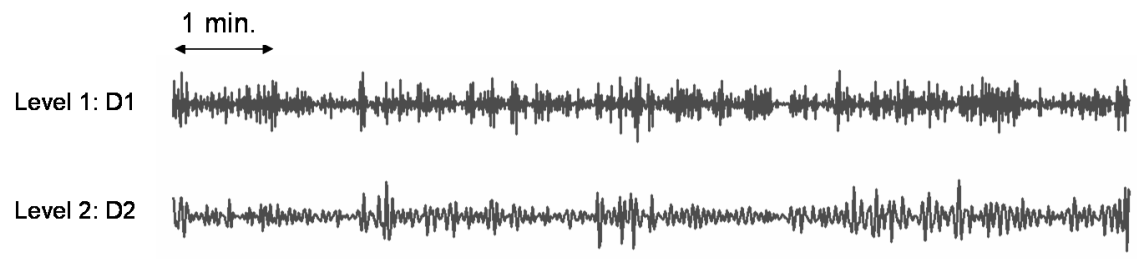

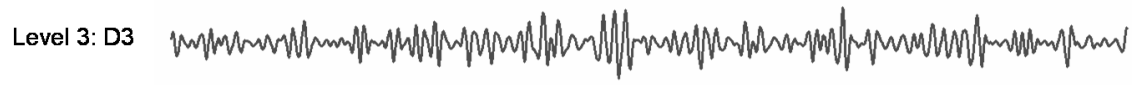
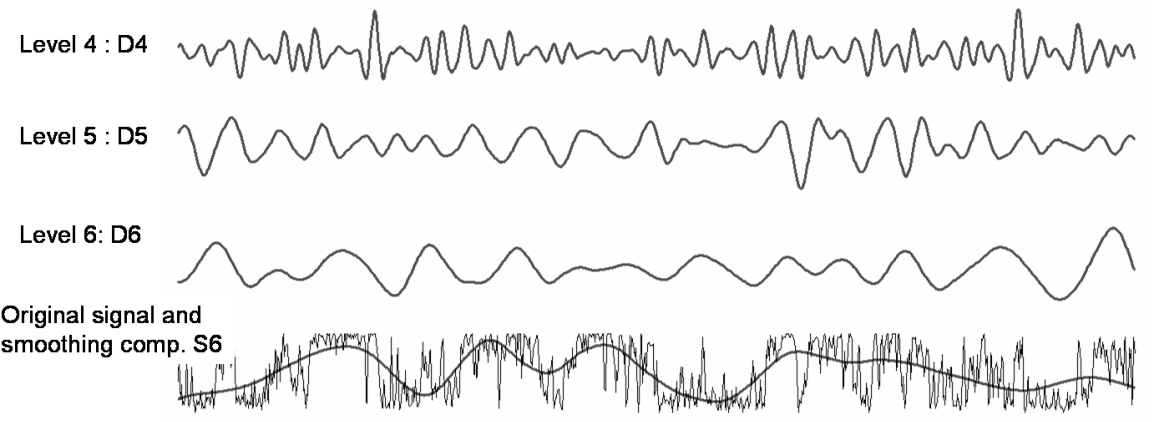

Fig. 4. Results of MRA decompositions with a signal length of 10 minutes. The bottom panel shows the original signal and smooth $A_{6}$ component.

component from the decomposed signals defines the baseline level, whereas the detailed components reveal the activities of the slow waves. On the other hand, artifacts and undesirable signals due to disturbing factors involve high frequencies, or are represented by detailed components at low scale levels. Therefore, in term of the energy preservation, (2) can be rewritten as:

$$
\|E\|^{2}=\sum_{j=1}^{k} D_{j}^{2}+\sum_{j=k+1}^{J} D_{j}^{2}+S_{J}^{2}
$$

where $\sum_{j=1}^{k} D_{j}^{2}$ can be filtered out from the original signal. When amount $\sum_{j=k+1}^{J} D_{j}^{2}$ is large enough, the signal after noise elimination is superimposed on the baseline level $S_{J}^{2}$. Therefore, it denotes a peak that can be considered as a waveform of the slow waves. For example, in Fig. 5a with $k=2$ defined noise levels, the peaks within a duration of $\Delta T=1$ min. exceeded the smoothing component at level 6. Results of the peak detection along a CE transit time of 1 hour are plotted in Fig. $5 \mathrm{~b}$.

\subsection{Evaluating the Propagation of Slow Waves}

Intuitively, the appearance of the waveforms at adjacent locations indicates the propagation of slow waves. The results in Sec. 3.2 allow for us to count the 


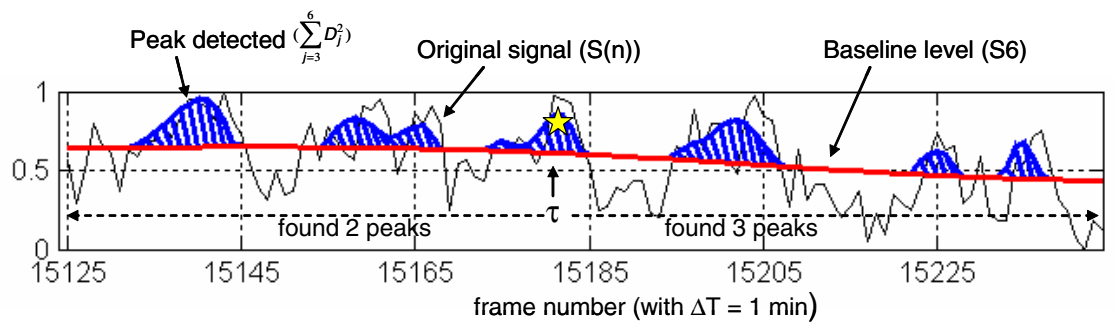

(a) Waveform detection at 1 min. around [A] (with detail components of scales 1 and 2 denoting noise and smoothing component at level 6 denoting a baseline)

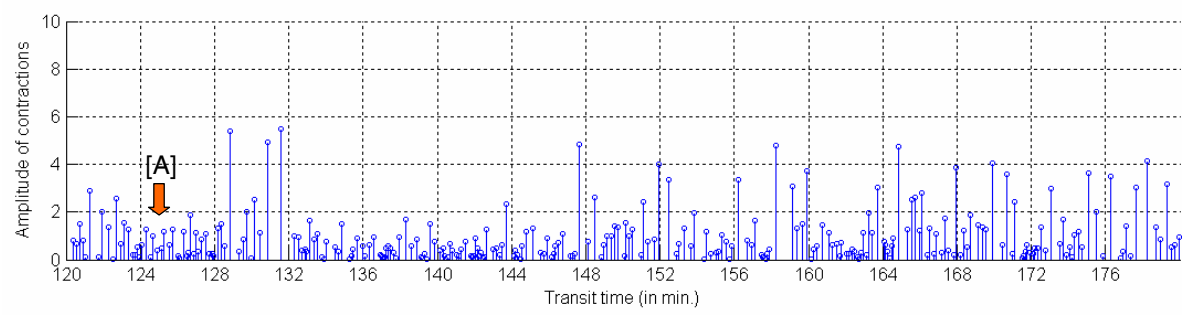

(b) Results of the waveform detection at 1 hour

Fig. 5. Waveform detection by the decomposed signals

appearance of waveforms around their neighbors. For example, the number of waveforms around the indicated peak (marked by yellow-star) in Fig. 5a is 6 (before $30 \mathrm{sec}$. and after $30 \mathrm{sec}$.). This means that the frequency of the slow waves at the selected position $\tau$ is $f_{s w}=6$ cycles $/ \mathrm{min}$. In fact, within a certain period of time $\Delta T$, there are several values of $f_{s w}$ because it yields from various waveforms within $\Delta T$. Therefore, a probability of waveform appearances relying on the number of observations at a certain $f_{s w}$ is calculated by:

$$
P_{f_{s w}^{i}}=\frac{C\left(f_{s w}^{i}\right)}{\sum(\text { peaks detected })},
$$

with $\mathrm{C}($.$) counting waveform appearances at f_{s w}$ and $i=1$ to $\max \left(f_{s w}\right)$ within time $\Delta T$.

The propagation of slow waves can also be measured by the velocity of each waveform along the $\mathrm{CE}$ transit time. If a waveform length is spread across $\delta t$ sec., and the accumulation of the CE displacements within $\delta t$ is denoted by $\Delta d$, then the velocity of the slow waves is defined by:

$$
v_{t}=\frac{\Delta d}{\delta t}(\mathrm{pixel} / \mathrm{sec})
$$

$\operatorname{Motion}(n)$ is a reasonable feature to calculate $\Delta d$. Figure $6 \mathrm{a}$ and Fig. $6 \mathrm{~b}$ show the propagation of the waveforms estimated by (5) within 1 minute in the proximal and distal regions, respectively. While Fig. 6a shows high propagation because of the short time in each waveform, Fig. 6b shows slower propagation. 


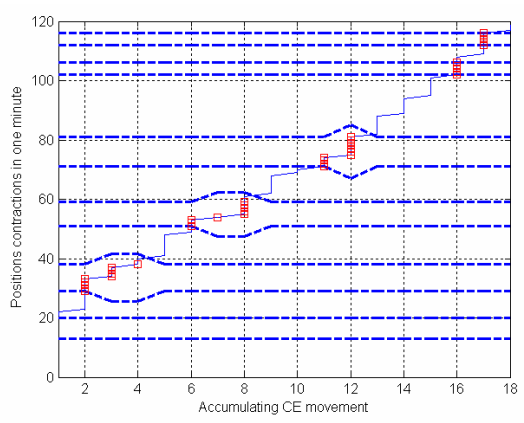

(a)

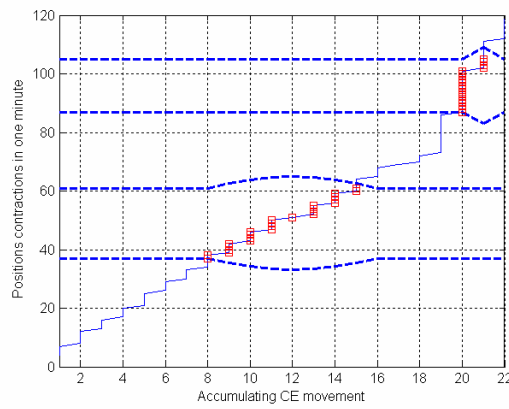

(b)

Fig. 6. Propagation of the waveforms within $1 \mathrm{~min}$. in the proximal (a) and distal (b) regions. The solid lines show the accumulation of CE displacements. The dashed lines indicate the position of waveforms. Squares mark CE movement within a waveform.

\section{Experimental Results}

To test the proposed method, sequences were obtained from three healthy volunteers, which was supported by the Graduate School of Medicine, Osaka City University. These sequences were examined and the frames showing the digestive organs such as the first gastric, the first duodenum and the first ileum were marked by the endoscopist experts. Table 1 shows data used in the experiments. The procedures used to extract the image features in Sec 2.2 were implemented by a $\mathrm{C}++$ program on a PC Pentium $3.2 \mathrm{GHz}, 1 \mathrm{~GB}$ Ram. Wavelet Packet Toolbox in MatLab was used to implement the MRA technique in Sec. 3.1.

Table 1. The material in experiments

\begin{tabular}{lccccll}
\hline Seq. & $\begin{array}{c}\text { Total } \\
\text { frames }\end{array}$ & $\begin{array}{c}\text { The first frame } \\
\text { of duodenum }\end{array}$ & $\begin{array}{c}\text { The first frame } \\
\text { of ileum }\end{array}$ & Total time & $\begin{array}{c}\text { Time in Small } \\
\text { bowel (in min.) }\end{array}$ \\
\hline Seq_1 & 60392 & 3161 & 31798 & $8: 23: 16$ & 238 \\
Seq_2 & 62350 & 7095 & 32863 & $8: 39: 35$ & 214 & \\
Seq_3 & 45020 & 3200 & 29037 & $6: 15: 10$ & 215 & \\
\hline
\end{tabular}

Figure 7 shows the results of Seq_1. The left panel plots ribbons that shows frequency components $\left(f_{s w}\right)$ along the CE transit time with predetermined configurations as described in Sec 3.2 . Examining the ribbons of $f_{s w}$, we can see high probability at the end of the small bowel for low frequencies and with the contrasting observations for high frequencies. More clearly, Fig. 7b shows the probability (calculated by (41) ) of low $f_{s w}$ values $\left(f r e q_{1} \leq f_{s w} \leq f r e q_{2}\right)$ in the upper panel and high $f_{s w}$ values in the lower panel. The right panel in Fig. 7a plots the velocities of slow waves, as calculated by (5). A simple fitting model was used to estimate the trends of velocities. The trend shows that the velocity of slow wave propagation gradually reduces along the CE transit time. Similar 

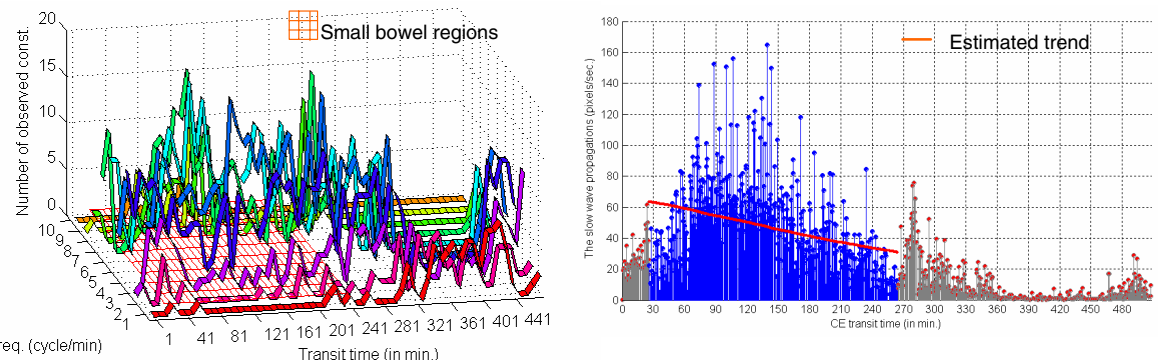

(a) Left panel: Ribbons of $f_{s w}$ components along the CE transit time. Right panel: The velocities calculated by (5). The slant line represents an estimated trend of the velocity in the small bowel regions (marked by stems) using a least square fitting model.
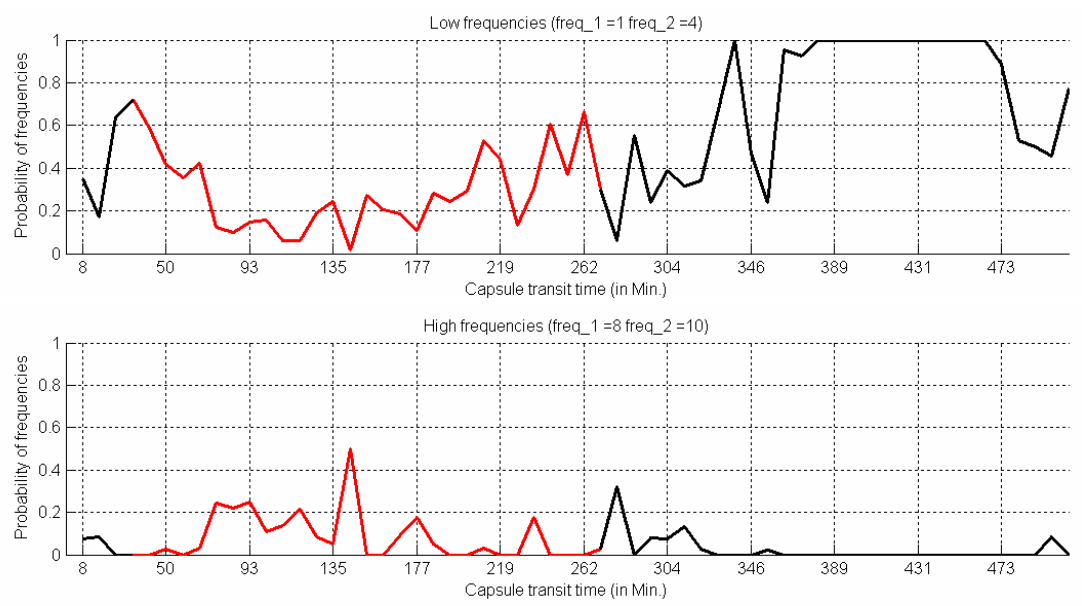

(b) Upper panel with $\mathrm{freq}_{1}=1 \mathrm{cycle} / \mathrm{min} \leq f_{\mathrm{sw}} \leq f r e q_{2}=4 \mathrm{cycles} / \mathrm{min}$. Lower panel with $f r e q_{1}=8 \mathrm{cycles} / \mathrm{min} \leq f_{\text {sw }} \leq f r e q_{2}=10 \mathrm{cycles} / \mathrm{min}$

Fig. 7. Slow wave propagation of Seq_1
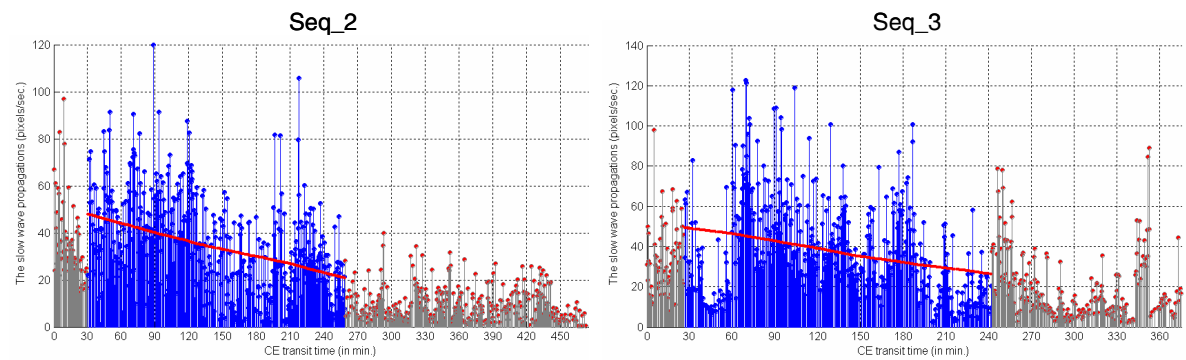

Fig. 8. Slow wave propagation of Seq_2 and Seq_3 
results are also shown in Fig. 8 for Seq_2 and Seq_3. By examining the three sequences, the propagation of slow waves interpreted by the proposed method is a good match with well-known characteristics of slow waves, as stated in [12 3 .

\section{Discussions and Conclusions}

This study was to investigate of the small intestinal motility using CE image sequences. In fact interest in small intestinal motility had also measured by other techniques such as in 4] or in [20121]. The proposed method thus needs to be confirmed by these measurements using same healthy control data. On the other hand, the proposed method still lacks evidence and suffers ambiguous explanations for noise and other factors for separation from the decomposed signals. The relationship between the segmentary contractions and smoothing components can support the definition of the baseline level as well as verifying the waveform detection results. In terms of experimental data, the frequency patterns estimated from patient data, which represent dysfunctional motility can offer more valuable evaluation.

In summary, this paper proposed a heuristic method using functional signals extracted from CE image sequences to study intestinal motility. The functional signal was averaged from multiple image features, by observing the high correlations between them. To exploit the information content of the functional signal, a MRA technique in wavelet domain was used. MRA results allowed us to separate the noise and the slow wave signal. The slow wave waveforms were detected when their energy exceeded the baseline level. Some analyses relied on the frequency appearance of the waveforms and their velocity along CE transit time were evaluated to estimate the propagation of slow wave. The experimental results shown provide the first results of a detailed interpretation of intestinal motility from CE image sequences. Therefore, we believe that this provides a promising method with which to develop clinical applications.

\section{References}

1. Grundy, D.: GastroIntestinal Motility - The Integration of Physiological Mechanisms. MTP Press Limited, Lancaster (1985)

2. Bronzino, J.D.: The Biomedical Engineering Handbook, 3rd edn. CRC Press, Boca Raton (2006)

3. Dunn, R.B., Linkens, D.A.: A mathematical model of the slow-wave electrical activity of the human small intestine. Medical and Biological Engineering 12, 750-758 (1974)

4. Hansen, M.B.: Small intestinal manometry. Physiological Research 51, 541-556 (2002)

5. Iddan, G., Meron, G., Glukovsky, A., Swain, P.: Wireless capsule endoscope. Nature 405, 417 (2000)

6. Adler, D.G., Gostout, C.J.: Wireless capsule endoscopy - state of art. Hospital Physician, 14-22 (2003) 
7. Swain, P., Fritscher-Ravens, A.: Role of video endoscopy in managing small bowel disease. GUT 53, 1866-1875 (2004)

8. Spyridonos, P., Vilarino, F., Vitria, J., Azpiroz, F., Radeva, P.: Identification of intestinal motility events of capsule endoscopy video analysis. In: Blanc-Talon, J., Philips, W., Popescu, D.C., Scheunders, P. (eds.) ACIVS 2005. LNCS, vol. 3708, pp. 531-537. Springer, Heidelberg (2005)

9. Spyridonos, P., Vilarino, F., Vitria, J., Azpiroz, F., Radeva, P.: Anisotropic feature extraction from endoluminal images for detection of intestinal contractions. In: Larsen, R., Nielsen, M., Sporring, J. (eds.) MICCAI 2006. LNCS, vol. 4191, pp. 161-168. Springer, Heidelberg (2006)

10. Vilarino, F., Spyridonos, P., Vitria, J., Azpiroz, F., Radeva, P.: Linear radial patterns characterization for automatic detection of tonic intestinal contractions. In: Martínez-Trinidad, J.F., Carrasco Ochoa, J.A., Kittler, J. (eds.) CIARP 2006. LNCS, vol. 4225, pp. 178-187. Springer, Heidelberg (2006)

11. Vu, H., Echigo, T., Sagawa, R., Yagi, K., Shiba, M., Higuchi, K., Arakawa, T., Yagi, Y.: Contraction detection in small bowel from an image sequence of wireless capsule endoscopy. In: Ayache, N., Ourselin, S., Maeder, A. (eds.) MICCAI 2007, Part I. LNCS, vol. 4791, pp. 775-783. Springer, Heidelberg (2007)

12. Vu, H., Echigo, T., Sagawa, R., Yagi, K., Shiba, M., Higuchi, K., Arakawa, T., Yagi, Y.: Adaptive control of video display for diagnostic assistance by analysis of capsule endoscopic images. In: Proc. of the 18th ICPR, pp. 980-983 (2006)

13. American Society for Gastrointestinal Endoscopy - ASGE: Technology status evaluation report wireless capsule endoscopy. Gastrointestinal Endoscopy 56, 1866-1875 (2002)

14. Swain, M., Ballard, D.: Color indexing. International Journal of Computer Vision 7, 11-32 (1991)

15. Lucas, B.D., Kanade, T.: An iterative image registration technique with an application to stereo vision. In: Proc. of the Intl. Joint Conf. on Artificial Intelligence, pp. 674-679 (1981)

16. Tomasi, C., Kanade, T.: Detection and tracking of point features. Technical report (1991)

17. Birchfield, S.: KLT: Kanade-Lucas-Tomasi Feature Tracker (2006), http://www.ces.clemson.edu/ stb/klt/

18. Canny, J.: A computational approach to edge detection. IEEE T-PAMI 8, 679-698 (1986)

19. Percival, B.D., Walden, T.A.: Wavelet Methods for Time Series Analysis. Cambridge University Press, Cambridge (2000)

20. Schemann, M., Ehrlein, H.-J.: Computerised method for pattern recognition of intestinal motility: functional significance of the spread of contractions. Medical and Biological Engineering 23, 143-149 (1985)

21. De Ridder, W.J.E., Voeten, J.J., Rombouts, J.A.C.A., Van Nueten, J.M., Schuurkes, J.A.J.: Computer-assisted method for analysis of postprandial gastrointestinal motility in conscious dogs. Medical and Biological Engineering 27, 470-476 (1989) 